\title{
Hunting the quicksilver: Using textual news and causality analysis to predict market volatility
}

\author{
Ameet Kumar Banerjee ${ }^{a, *}$, Andreia Dionisio ${ }^{b}$, H.K. Pradhan ${ }^{c}$, Biplab Mahapatra ${ }^{\text {a }}$ \\ ${ }^{\text {a }}$ Xavier Institute of Management Xavier University, Bhubaneswar, Odisha 751 013, India \\ ${ }^{\mathrm{b}}$ University of Evora, CEFAGE School of Social Sciences, Management Department, Largo dos Colegiais, 2, 7000, Evora, Portugal \\ ${ }^{c}$ XLRI -Xavier School of Management Jamshedpur, Jharkhand 831001, India
}

\section{A R T I C L E I N F O}

\section{Keywords:}

Sentiment scores

Bond markets

Information theory

Volatility

\begin{abstract}
A B S T R A C T
This paper proposes that the dynamics of bond volatility may be understood by studying textual news sentiments. In this new approach, a modified framework is used to understand the atypical characteristics of bond market news. The paper proceeds in two steps. First, a word list of sentiment terms is generated using three sentiment word lists to determine negative and positive news sentiment scores. Second, four measures of volatility are estimated and combined with a nonlinear technique adapted from information theory to understand the correlation and direction of causality between sentiment scores and measures of volatility. This paper shows that sentiments extracted from textual news published in the newspapers can explain bond returns volatility or the quicksilver. The empirical results support that news sentiment is highly correlated with the measures of volatility and that information flows unidirectionally from news to volatility. This study, perhaps the earliest work in text mining to examine the run of causality between news signals and bond return volatility, adapts a nonlinear technique from information theory to describe the nonlinear behavior of Indian debt markets and understand the volatility dynamics of the benchmark bond.
\end{abstract}

\section{Introduction}

News releases-macroeconomic, financial, or political—work as signals that can move financial markets. Policymakers and market participants continually monitor the news for macroeconomic signals and information in real-time. When the news is novel, different asset classes overreact significantly (Altavilla, Giannone, \& Modugno, 2017; Banerjee \& Pradhan, 2021; Banerjee, Pradhan, Tripathy, \& Kanagaraj, 2020; Evans, 2011; Luss \& d'Aspremont, 2015). Many studies analyze the link between news and asset prices (Elder, Miao, \& Ramchander, 2012; Miao, Ramchander, \& Zumwalt, 2014). A newer stream of research into the dynamics of asset pricing behavior analyzes the vast quantities of unstructured textual data available at online repositories of textual news like Facebook, Twitter, and Stocktwits to reveal newer perspectives (Bartov, Faurel, \& Mohanram, 2018; Tetlock, Saar-Tsechansky, \& Macskassy, 2008); empirics feel that analyzing the news content in these raw, unstructured datasets may help uncover the intrinsic sentiments mostly absent in quantitative datasets.

Textual analysis has been used to understand the tonal content of the news (positive vs negative emotions) and demystify their impact on financial markets and prices (Tetlock, 2007; Tetlock et al., 2008). Loughran and McDonald (2011) use earnings-related words and phrases from the public filing information with the US Securities and Exchange Commission (SEC) and establish that these impact stock returns. Bartov et al. (2018) report that Twitter helps in reducing information asymmetry and it can predict firm earnings. Studies use textual news to discover the impact of corporate disclosures (Price, Doran, Peterson, \& Bliss, 2012; Twedt \& Rees, 2012); textual sentiments on the performance of initial public offerings (IPO) (Ferris, Hao, \& Liao, 2013; Loughran \& McDonald, 2011); the relationship between internet board postings and trade volumes (Das \& Chen, 2007); investor sentiments in Stocktwits messages on the returns on stocks and cryptocurrencies (Chen, Despres, Guo, \& Renault, 2019; Gan, Alexeev, Bird, \& Yeung, 2020); and the effect of firm specific textual news on trade and volatility (Boudoukh, Feldman, Kogan, \& Richardson, 2019; Caporin \& Poli, 2017).

The response of structured news arrivals on asset price volatility has been studied as well (Banerjee et al., 2020; Banerjee \& Pradhan, 2020; Campbell, Lettau, Malkiel, \& Xu, 2001; Elder et al., 2012; McNeil \& Frey, 2000), but little is known about the impact of textual news on the second moment of returns in debt instruments; Gotthelf and Uhl (2019)

\footnotetext{
* Corresponding author.

E-mail addresses: ameet@ximb.edu.in (A.K. Banerjee), andreia@uevora.pt (A. Dionisio), pradhan@xlri.ac.in (H.K. Pradhan).
} 